\title{
Multi-Fuel Allocation for Power Generation Using Genetic Algorithms
}

\author{
Anurak Choeichum, Narongdech Keeratipranon* and Chaiyaporn Khemapatapan
}

\author{
College of Innovative Technology and Engineering, Dhurakij Pundit University, Bangkok, Thailand
}

\begin{abstract}
The ever increasing growth of energy consumption has stimulated an energy crisis, not only in terms of energy demand, but also the impact of climate change from greenhouse gas (GHG) emissions. Renewable energy sources (RES) have high potential toward sustainable development, with a wide variety of socioeconomic benefits, including diversification of energy supply and creation of domestic industry. This paper presents a solution to optimal multi-fuel allocation for the electric power generation planning problem via genetic algorithms (GA). The objective is to maximize the electric power energy output and minimize generation cost. This is a difficult problem because of its data variation and volatility. GA can provide an appropriate heuristic search method and return an actual or near optimal solution. This paper uses some heuristics during crossover and mutation for tuning the system to obtain a better candidate solution. An experimental result showed significantly improved results compared with other techniques. The results in this paper should be useful for connecting power generation with economic growth.
\end{abstract}

Keywords: Multi-fuel Allocation, Power Generation, Genetic Algorithms, Power Energy.

\section{INTRODUCTION}

Electricity is the most multi-purpose energy carrier in the modern global economy, and is linked to human and economic development (Aqeel and Gholamreza, 2011). The shortage and poor management of electrical energy stimulates energy crises throughout the world.

Renewable energy is widespread and is effectively developed throughout the world. Many renewable energy fuels, such as wind turbines and solar farming are among the most popular alternative energy sources (Warsono et al., 2007). However, the optimal management scheme of biofuel has not been studied extensively. This topic includes the optimal size and location of a power plant (Sedighizadeh et al., 2013), the optimal sizing of generation (Ying-Yi and RuoChen, 2012), types of raw agricultural materials, and efficient processes of energy conversion technology (ECT), (Yang et al., 2016). Proper allocation of a raw material will have a high impact on the volume of produced electricity. Many countries in Asia are agricultural countries, with plenty of biofuel materials. Currently, over ten thousand tons of local agricultural materials are used to produce thousands of Megawatts (MW) of electricity (Twarath, 2012). However, with plenty of raw materials, Asia can produce bio-energy more than ten thousand of Megawatts (Japan Institute of Energy, 2014).

*Address of correspondence to this author at the College of Innovative Technology and Engineering, Dhurakij Pundit University, Bangkok, Thailand; Tel: +66 2954-7300; Fax: +66 2954-7356;

E-mail: narongdech.ken@dpu.ac.th

JEL: L71, Q01, Q16.
Optimal power generation planning has been considered frequently using classical optimization methods, namely as the maximization of an objective function representing energy (Sedighizadeh et al., 2013) or the minimization of an objective function representing generation cost (Amirsaman et al., 2014), (Christian et al., 2008), (Jorg et al., 2010), (Senjyu et al., 2006). The Genetic Algorithm (GA) offer a powerful approach to these optimization problems, made possible by the increasing availability of high performance computers. GA is a parallel and global search technique that emulates natural genetic operators.

GA operates on a population of candidate solutions encoded in a finite set of bit strings, called chromosome. In order to obtain optimality, each chromosome exchanges the data by operators borrowed from natural genetics to produce a better solution.

In order to have high productivity, a proper match between a raw material and an ECT is required. Some raw material might be suitable with one technology, but not the other. This paper searches for an optimal method to match the raw material with the technology for the highest productivity, along with the proper volume to suit daily prices.

This paper enables the use of genetic algorithms to improve bio-energy allocation in biofuel power plants. It can analyze the proper types of fuels for each ECT, along with the proper production volume for each day, to maximize the produced electricity value. The power generation scheme can be planned quickly and 


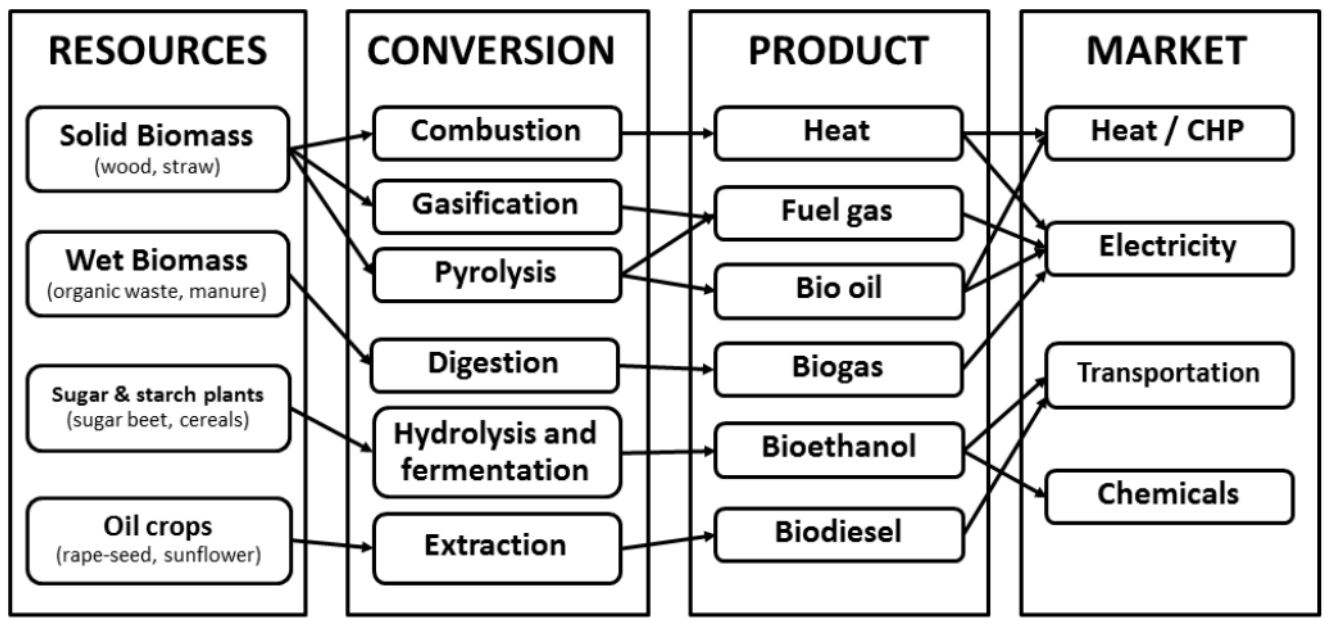

Figure 1: Energy Conversion Technology.

efficiently according to the quantity and types of available fuels. This will increase the stability of the power system, enhancing regional and rural development opportunities.

\section{PROBLEM FRAMEWORK}

\subsection{Challenge}

There are several options to produce electricity from biomass (Sedighizadeh et al., 2013), (Fakhimghanbarzadeh et al., 2010). Solid and wet biomass are used as plant input feedstock, with ECT being the conversion process, as shown in Figure 1. This system must integrate many interrelated input data, including the raw material stock, availability of ECT, and volatility of daily electricity price. High electricity output can be obtained from a proper match between raw material inputs and ECT. For example, combustion technology is suitable for solid biomass,wood piece, not sugarcane plant. The electricity price in this paper is assumed to change daily, so that the system must plan to produce more on higher price days. Production is absolutely essential to consider or plan a period for maximizing profits.

\subsection{Input: Multi-Fuel}

Renewable energy sources (RES) are the input data of this system to convert fuel into electricity. The research simulates the inputs into five biomass fuels, which are rice husk, bagasse, wood pieces, straw, and corn cob. Each type of fuel has different properties, such as energy efficiency, heating value, and moisture content, and will be stored in inventory for raw material reserved in the power plant. Biofuel management must be carefully planned, including the sourcing of the raw material, delivery, storage, usage plan, and garbage management. Moreover, the use of biomass can contribute to sustainable development in rural areas (Lijun et al., 2008).

\subsection{Processing Unit: Energy Conversion Technology (ECT)}

The ECT is the processing unit for energy production of the system, and will produce electricity from multi-fuel inputs. This research has four different ECT, which are combustion technology, gasification technology, pyrolysis technology, and digestion technology, as shown in Figure 2.

\subsection{Output: Electricity Produced}

The produced electricity is the output of the system. Before the fuel is fed to the ECT, this system will evaluate energy efficiency processing and find the best time to produce and sell electrical energy.

\section{GENETIC ALGORITHM IN OPTIMAL FUEL ALLOCATION}

\subsection{Overview}

In this paper, we develop a GA applied to the problem of optimal multi-fuel allocation for electric power generation planning as shown in Figure 3. The research was conducted in a simulation with three different factors, namely five types of raw materials, four types of ECT, and the daily electricity price.

\subsection{Chromosomes and Genes}

A representation of a possible solution to an optimization problem is encoded using either binary, 


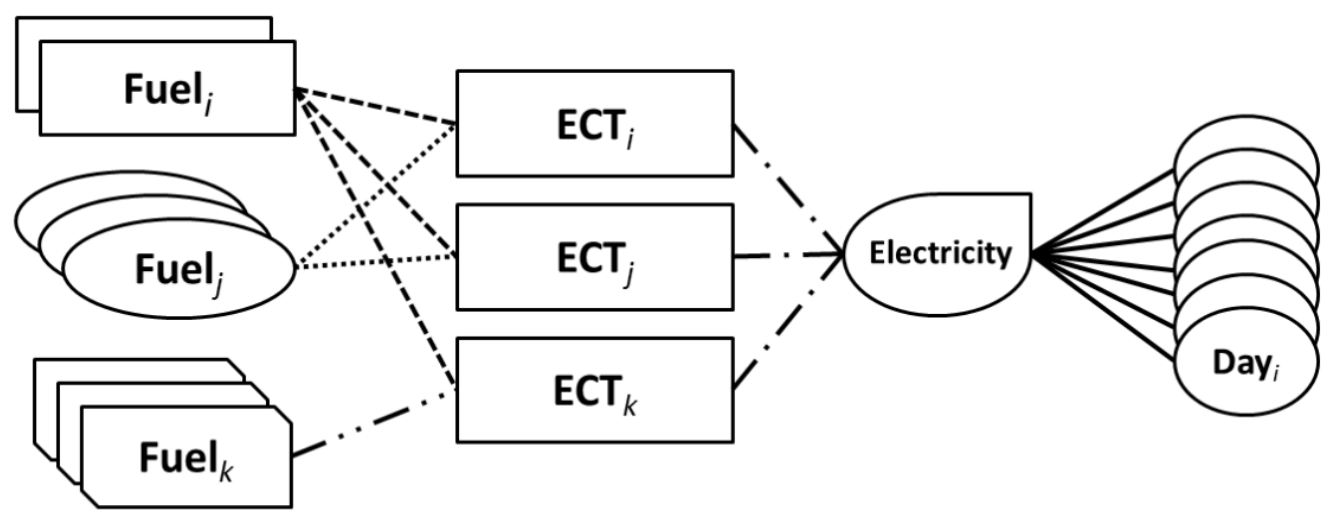

Figure 2: Overview of Solution.

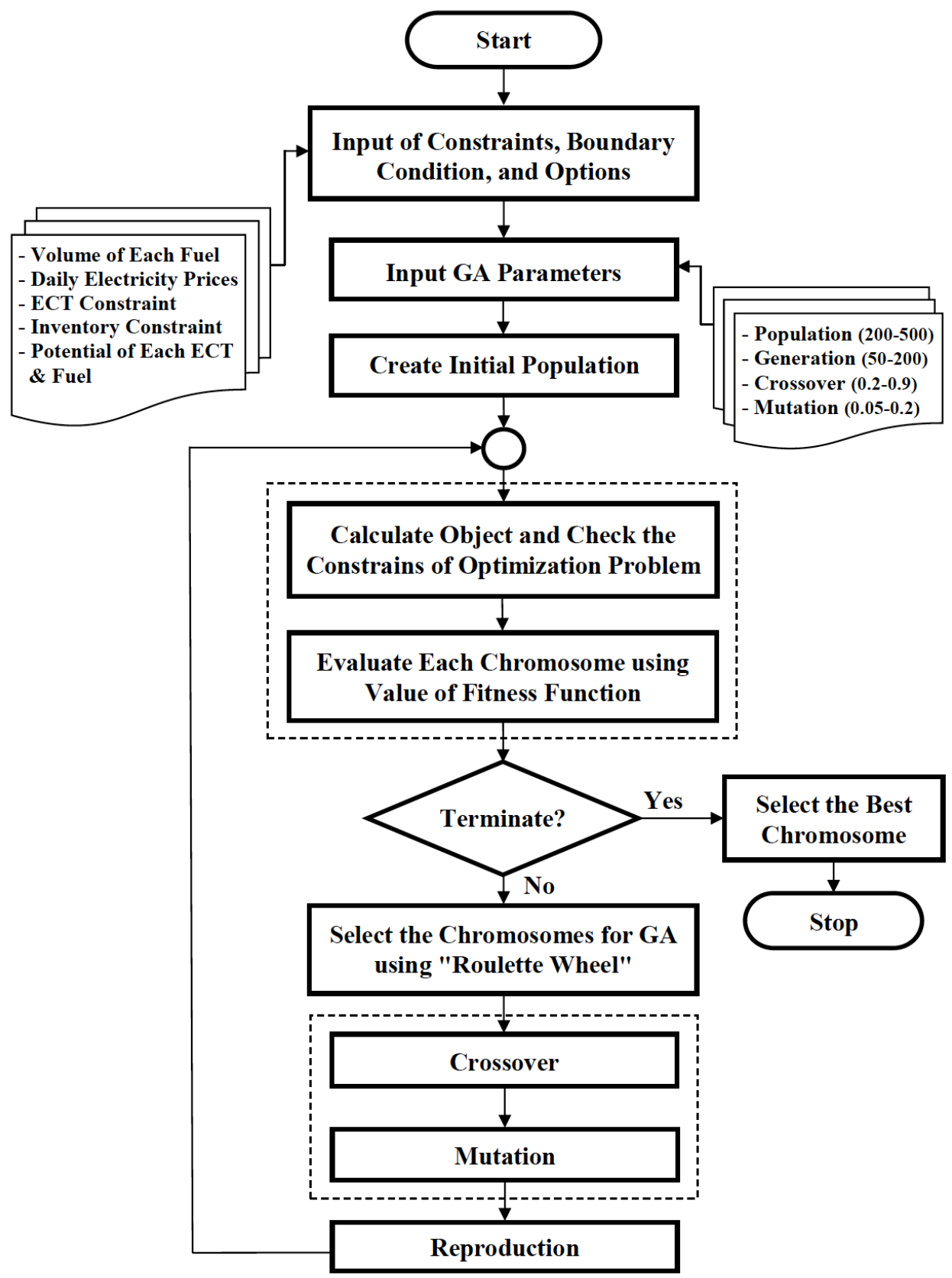

Figure 3: Flow Chart of Allocation using GA. 


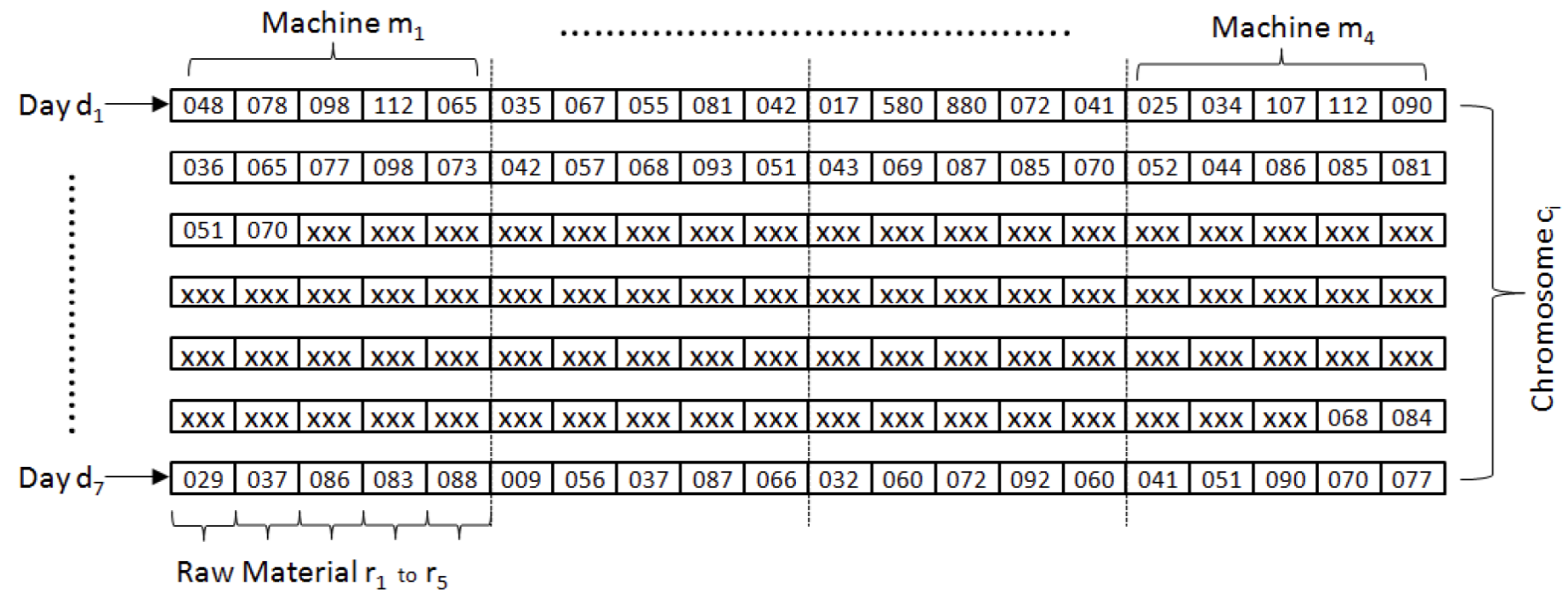

Figure 4: Conceptual Illustration and Components.

real-valued, or tree encoding. Gene is a group of bits or real values which encode a particular element of a possible solution (Chromosome). Each set of chromosomes represents an individual (Abdul et al., 2011), which is one possible solution to fuel allocation. If all constraints are satisfied, then it can be considered as a valid solution. Each chromosome represents four ECT, and each ECT stores five fuels in an array. The index of the array specifies the position of the raw material in the ECT.

The research has made a preliminary structural design of the system based on the GA. Each candidate solution is encoded in the form of chromosomes, as shown in Figure 4 . There is a maximum capacity of storage for raw materials in the inventory, as shown in equations (1) and (2), with the GA process evolving the value in the chromosomes to achieve a better candidate solution:

$\Sigma_{m \in M} G_{m t} \leq \operatorname{Cap}_{t}$,

$\Sigma_{m \in M} G_{m t} \leq$ Stook $_{m}+\left[\right.$ Supply $\left._{m}\right]$.

Each of the chromosomes in this paper has 7 days with 140 genes. There are four types of ECT, and five types of raw materials for each ECT. The daily electricity price changes regularly.

\subsection{Selection}

The calculated fitness of each chromosome helps to select suitable members for the next generation. This selection method is done using the roulette wheel technique (Abdul et al., 2011). Figure 5 shows the concept of the roulette wheel selection where size of each partition depends on its fitness value. The higher fitness value, the bigger partition, and hence the higher chance to be selected for the next generation. Although, a member with high fitness value tend to be selected, but a lower fitness member also has a chance to be selected as well according to a random value. This make a new generation more diversifies and is not totally dominated by the fittest member. To implement this idea, each member will have a probability to be selected, as shown in equation (3):

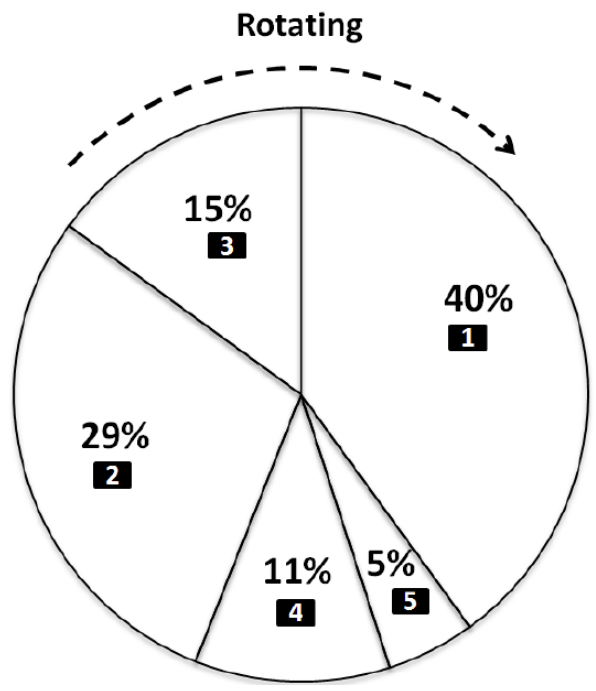

Figure 5: Roulette Wheel Selection.

$$
P_{k}=\frac{f_{k}}{\sum_{j=1}^{\text {popsize }} f_{j}},
$$

where

$P_{k}=$ selection probability;

$f_{k}=$ fitness value of member $\mathrm{k}$. 


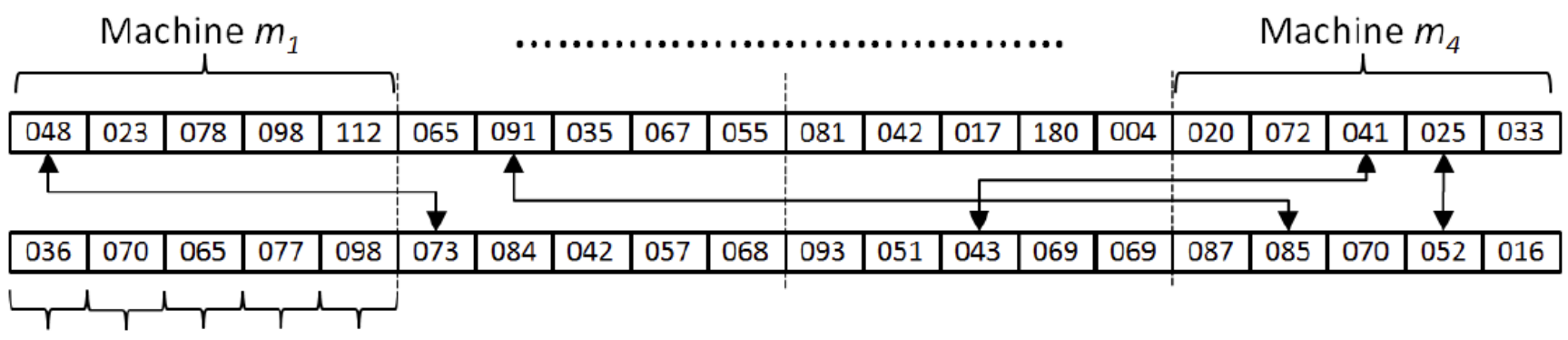

Raw Material $r_{1}$ to $r_{5}$

Figure 6: Crossover of Fuels.

\subsection{Crossover}

Crossover is the primary genetic operator which promotes the exploration of new regions in the search space (Younes et al., 2007), an operation in which genetic material is exchanged between two different chromosomes to generate new chromosomes or a child (Zhang et al., 2007). The crossover method takes information from one individual and inserts it in the other to create a new child (Abdul et al., 2011). The probability of crossover rating can be configured and modified. Figure 6 shows the crossover of fuel and Figure 7 shows the crossover of ECT.

\subsection{Mutation}

Mutation is a genetic operator used to maintain genetic diversity from one generation of a population of chromosomes to the next (Abdul et al., 2011). In other word, this operation is randomly changes parts of a gene in a chromosome at randomly chosen places. The mutation adds a random search to the GA (Zhang et al., 2007). The mutation technique is an evolved characteristic, and is strongly influenced by the genetics of each version. The variety of mutation techniques used to find a better result for this system include a mutation of fuels and mutation of ECT, as shown in Figures $\mathbf{8}$ and $\mathbf{9}$.

\subsection{Reproduction}

Reproduction is used to generate a next generation from those selected through genetic operators based on the principle of better fitness survival. It is an operator that obtains a number of solutions according to their fitness value. For each new solution to be produced, a pair of parent solutions is selected for breeding from the pool that was selected previously. By producing a child solution using crossover and mutation, a new solution is created, which typically shares many of the characteristics of its parents, and new parents are selected for each child. This process continues until a new population of appropriate size is generated.

\subsection{Fitness Function}

The fitness function can be described by equation (4), cash inflows:

$$
P V_{t}=P_{g} \cdot E_{g} \cdot\left[K g \cdot\left(\frac{1-K_{g}^{V u}}{1-K_{g}}\right)\right]
$$

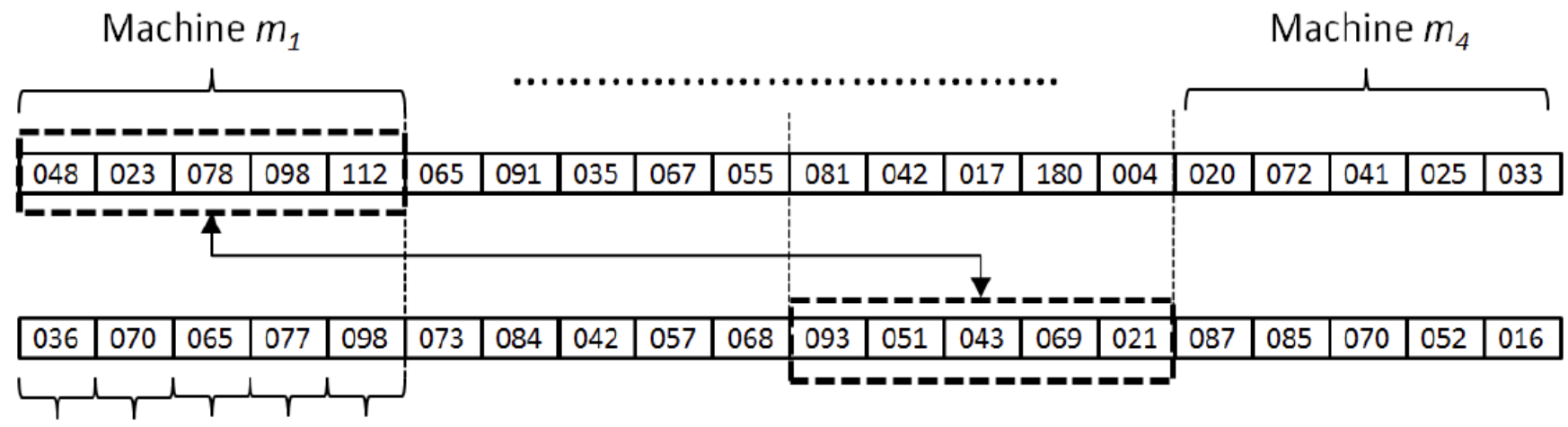

Raw Material $r_{1}$ to $r_{5}$

Figure 7: Crossover of Machines (ECT). 


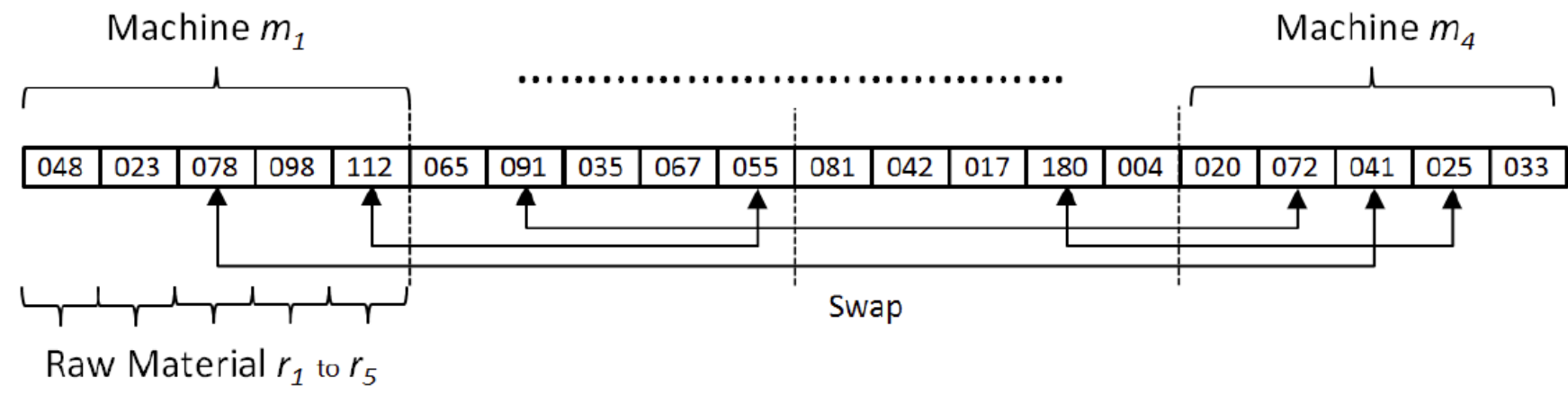

Figure 8: Mutation of Fuels.

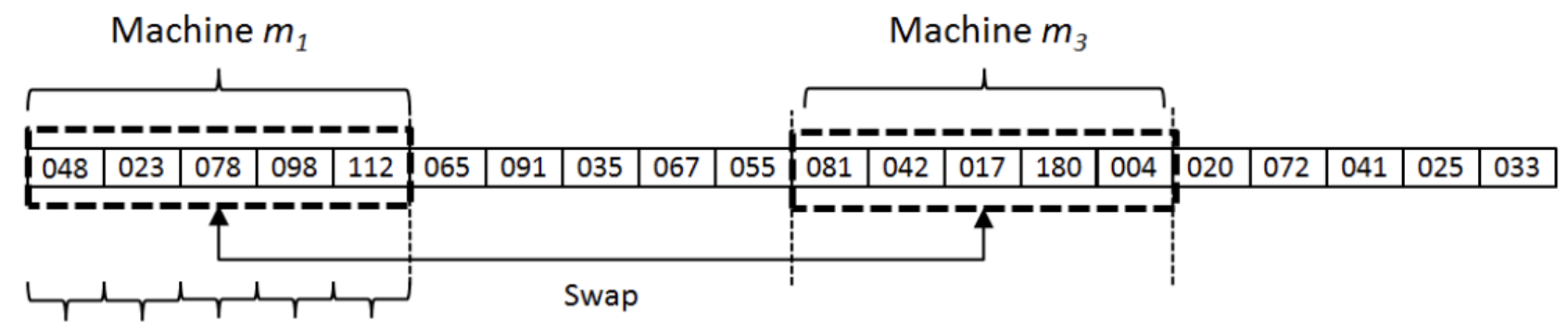

Raw Material $r_{1}$ to $r_{5}$

Figure 9: Mutation of Machines (ECT).

where

$P V_{t}=$ gain from the sold electric energy;

$P_{g}=$ selling price of electric energy;

$E_{g}=$ sold and produced electric energy;

$K_{g}=$ increasing rate of sold energy price;

$V_{u}=$ useful lifetime.

In order to choose which children from the newly created population will be favored to breed, the fitness of every individual has to be computed. In this paper, equations (5) and (6), is the fitness function of this implementation:

$\max \sum_{t=1}^{T} P_{t}$,

Fitness Function $=P_{t}=P V_{t}-\left[\operatorname{In} v_{s}+L P_{t}+M C_{t}\right]$,

where

$P_{t}=$ maximum of selling;

$I n v_{s}=$ investment cost in the production cycle;

$L P_{t} \quad=$ electricity used in manufacturing;

$M C_{t} \quad=$ maintenance technology.

\subsection{Termination of the GA}

As GA is a stochastic search method, it is difficult to specify convergence criteria formally. Termination is a criterion by which the GA decides whether to continue searching or to stop the search (Xiang et al., 2005), (Zhao et al., 2009). As the fitness of a population may remain static for a number of generations before a superior individual is found. A common practice is to terminate GA after a specified number of generations, and then test the fitness of best members in the last population (Younes et al., 2007). If no acceptable solutions are found, the GA may be restarted and a search initiated (Michalewicz, 1995). The process is repeated until a termination condition has been reached, as shown in equation (7). The condition is a combination between satisfying the maximum criteria and the maximum number of generations reached:

$\left.\begin{array}{c}\text { Iteration }_{t} \geq \text { Iteration }_{\max } \\ P_{t} \geq P_{\text {target }}\end{array}\right\}$ Termination Condition

\section{RESULTS}

The results shown in this section are obtained from various GA parameters, ' 1 ' is the 200 population size, ' 2 ' is the 500 population size $\left(' 1 '=200,{ }^{\prime} 2 '=500\right), T$ is the mutation rate of $10 \%, S$ is the mutation rate of $5 \%$ 


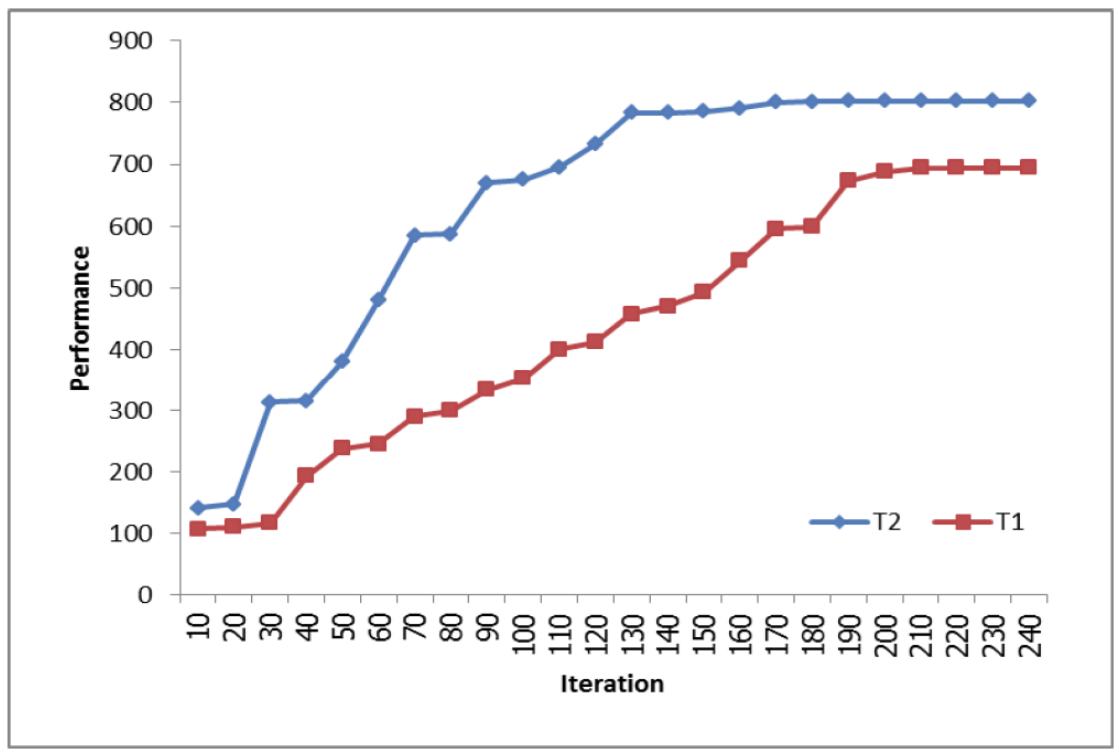

Figure 10: Comparison of Each Population Size.

( $T=10 \%, S=5 \%), V 1$ is the crossover of ECT, V2 is the mutation of fuel, and $V 3$ is the mutation of ECT. From this experiment, a GA with higher population size can find rapidly the best answer or using a fewer iteration. The results in Figure 10 show the 200 population size compared with the 500 population size, and the mutation rate of $10 \%$.

The results in Figure 11 show the 200 population size compared with the 500 population size and mutation rate of $5 \%$. Figure 12 shows the mutation rate of $10 \%$ and the mutation of fuel techniques.

Figure 13 shows the mutation between the ECT technique, 200 population size compared with 500 population size, and the mutation rate of $10 \%$, can describe the 500 population size and the mutation rate of $10 \%$. From this experiment, the mutation of ECT performs the best.

\section{DISCUSSION}

From the results, the larger population size is inversely proportional to the required iterations because the larger volume of the population can increase the chances of finding a better candidate. The mutation operation, each fuel has a probability to mutate. If a fuel is selected for mutation, the new value of the gene will be random within the range $+/-100 \%$ from its previous value. In this paper, a higher rate of

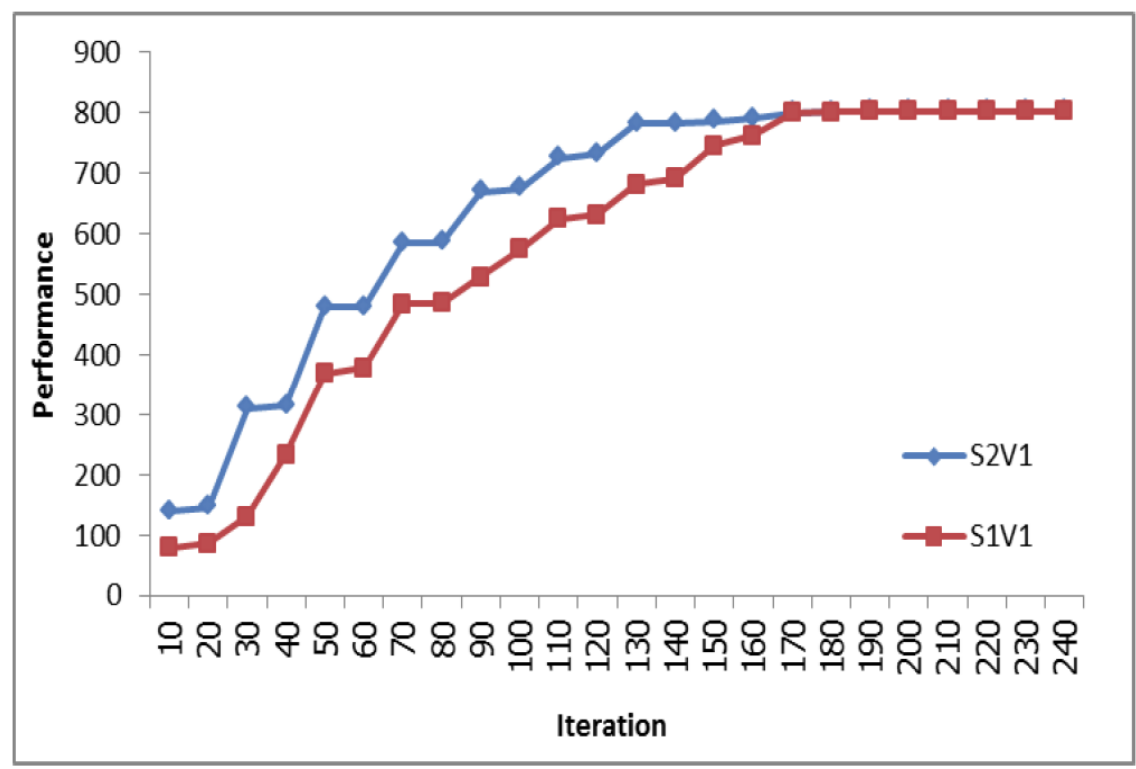

Figure 11: Comparison of Each Population Size and Crossover of Machines (ECT). 


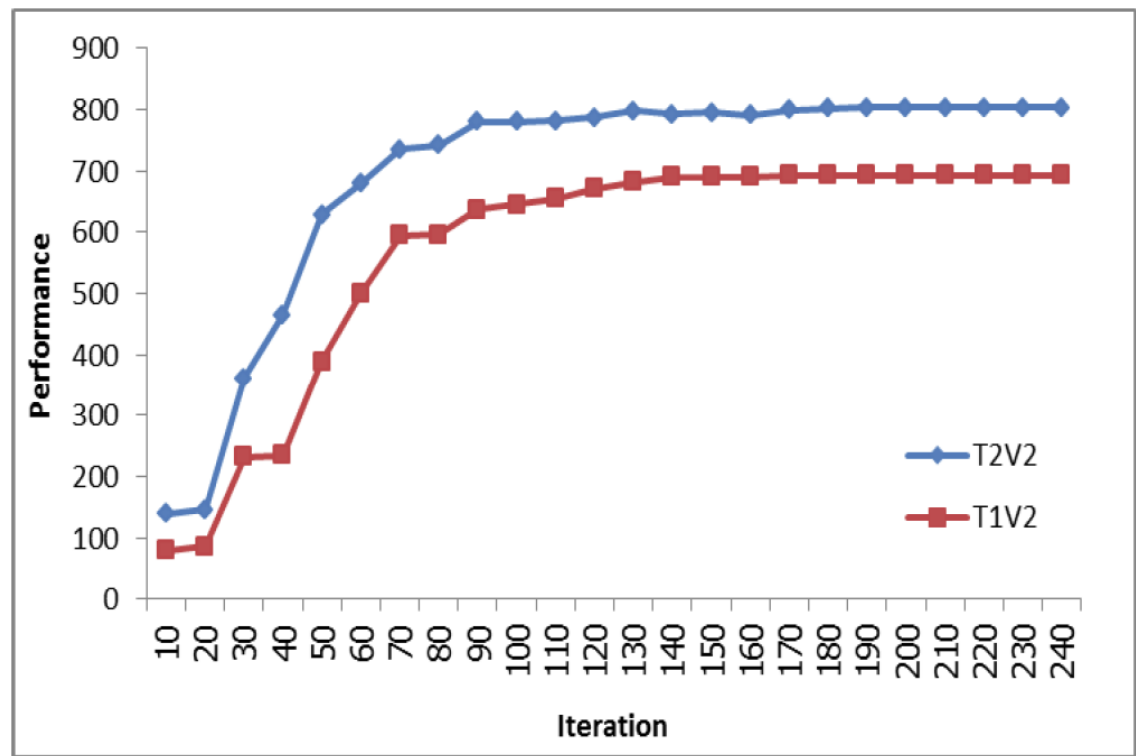

Figure 12: Comparison of Each Population Size and Mutation of Fuels.

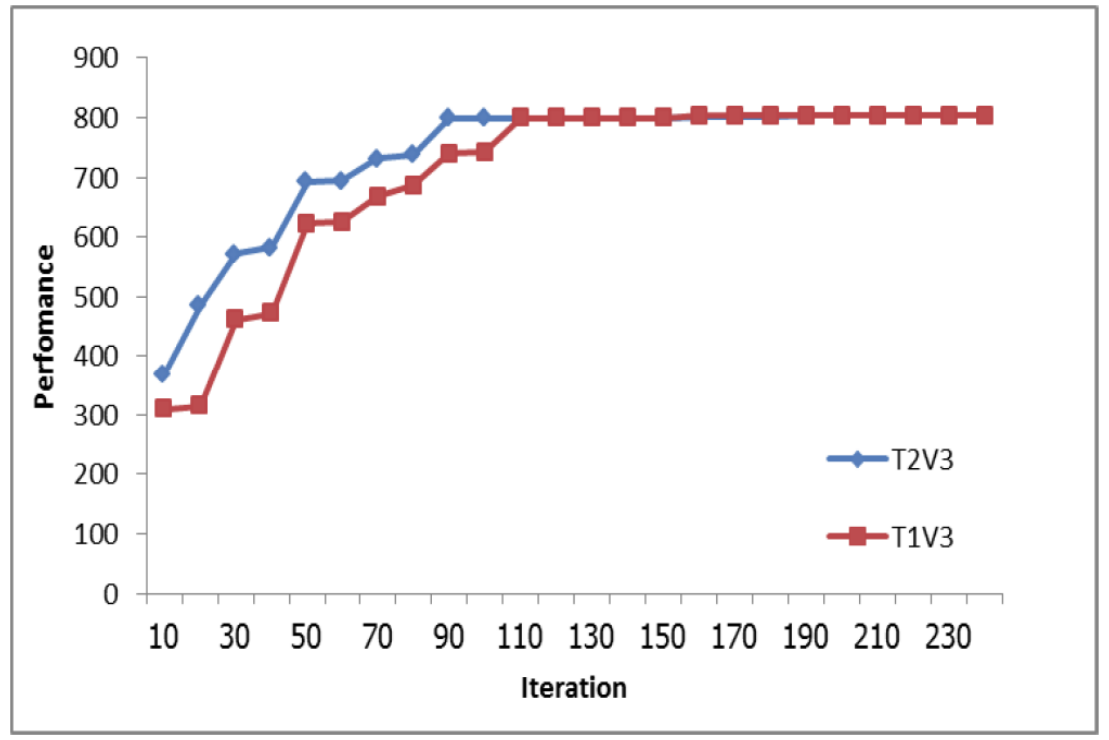

Figure 13: Comparison of Each Population Size and Mutation of Machines (ECT).

mutation leads to a quicker solution because its population used a new value more often than the original value. Both the crossover of ECT and the mutation of ECT are rapidly coverging at low generations.

The crossover and mutation operations have an impact on the performance of the GA. The crossover operation in a local exploration of the target function creates a combination of other individuals, while the mutation operation introduces parameter values into the gene, which have not been used previously.

Electricity prices increase rapidly, and the carbon credit issue is one of the most difficult problems to handle. Thus, the RES is the most popular choice for carbon reduction, but many RESs and the operational costs of renewable power plants are not affordable. Therefore, a suitable way to reduce GHG and the costs of RES power generation should be proposed.

In Figure 14, electricity prices in Denmark, Germany and Spain are the most expensive. Electricity in Denmark is mainly generated by diesel fuel. Canadian electricity is cheap at 10 US cents per kilowatt hour, which is reflected in their high average electricity usage. US electricity prices are $0.12 \$ / \mathrm{kWh}$, which is quite cheap. In India and China, they are also very cheap. The UK is in the middle at 20 cents. Electricity 


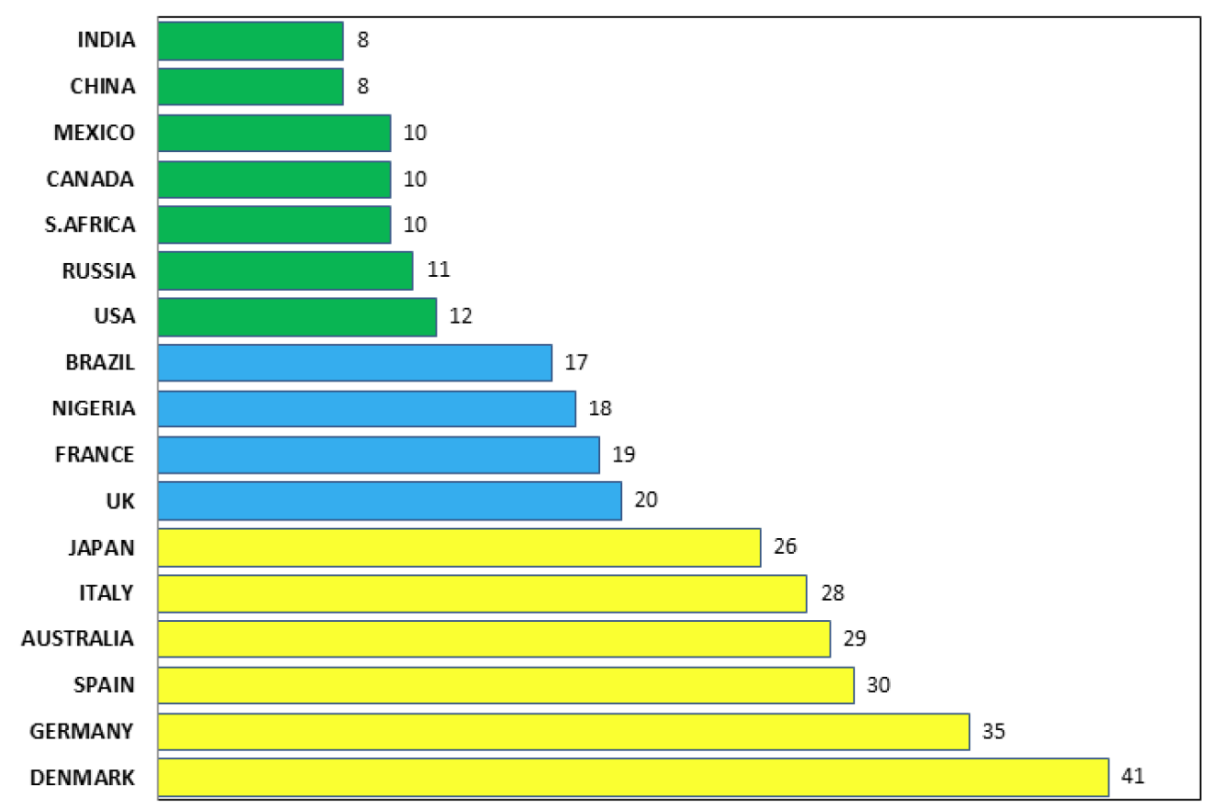

Figure 14: Average national electricity prices in US cents/kWh.

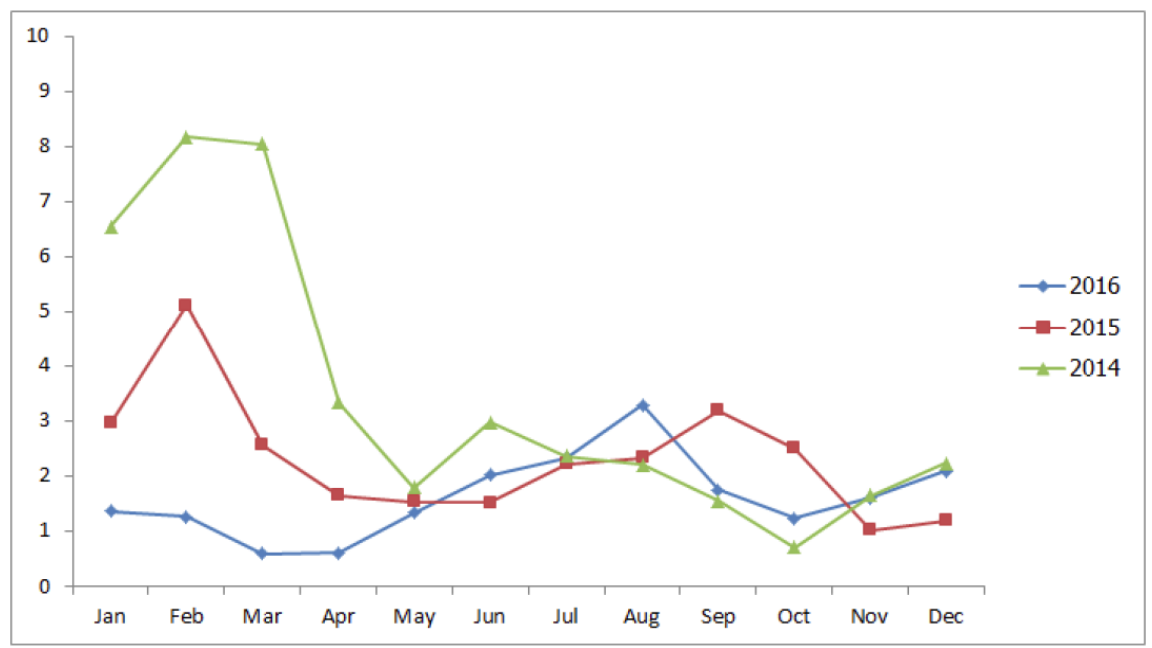

Figure 15: Monthly Prices.

is relatively expensive globally, but not too high for Europe, where most countries pay a high share of tax for their power. Electricity prices change many places, including South Africa, Australia and Nigeria (OVO Energy, 2015).

In addition, electricity prices also fluctuate each month, as shown in Figure 15. The rate of change for each month change automatically, depending on a number of factors.

Figure 16 shows daily changes in electricity prices (Can\$/MWh) in Ontario, Canada (Market Report, 2016). It was found to be volatile throughout the month and the price of electricity trading can be increased or decreased without limit. Electricity prices are always fluctuating, with high variability. Power generation planning is difficult, and may take a very long time to plan, evaluate, and calculate the multi-fuel allocation and electrical energy production. For this reason, it is necessary to develop tools or methods for improved allocation and planning.

\section{CONCLUSION}

This paper used genetic algorithm (GA) to allocate multi-fuel for power generation planning for the best solution. The aim of the paper was to maximize electric power energy and minimize generation costs. The research successfully demonstrated a method to provide suitable raw material allocation when there are differences in raw materials, energy conversion 


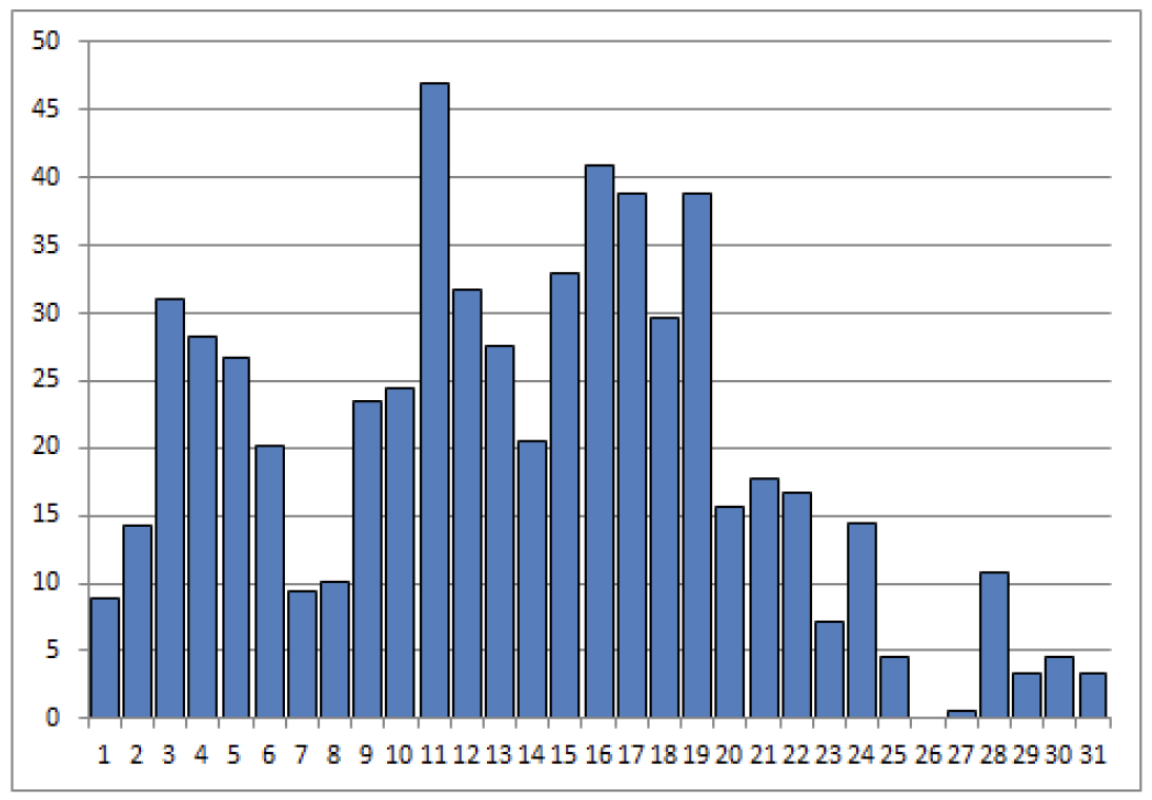

Figure 16: Daily Prices.

technologies (ECT), and volatility in electricity daily prices.

With this system, an investor can have a better allocation plan, and receive greater electricity output from the bio-energy power plant and highest returns. In response to the government power development plan, the paper can find optimal and sustainable solutions of complex problems for power generation. It can provide a solution for stability to the power grid, optimize energy conversion, and help satisfy decision-making requirements for maximum investment returns.

\section{ACKNOWLEDGEMENT}

The authors wish to thank Chia-Lin Chang and Michael McAleer for helpful comments and suggestions.

\section{REFERENCES}

Abdul K.M.M., Mohammad S., Faisal F., and Iqbal H.S. (2011), Solving the Vehicle Routing Problem using Genetic Algorithm, (IJACSA) International Journal of Advanced Computer Science and Applications, Vol.2, No. 7.

Amirsaman A., Mahmoud G., Mehdi E-A., Mohammed S.F. and Moein M-A. (2014), A Multi-Objective Transmission Expansion Planning Framework in Deregulated Power Systems With Wind Generation, IEEE Transactions on Power Systems, Vol. 29, No.6, November, 3003-3011. https://doi.org/10.1109/TPWRS.2014.2316529

Aqeel A. and Gholamreza Z. (2011), Sustainable Energy Systems: Role of Optimization Modeling Techniques in Power Generation and Supply - A review, ELSEVIER, Renewable and Sustainable Energy Reviews, 3480-3500

Christian W., Sean M., and Michael B. (2008), Biogas plant optimization using Genetic Algorithms and Particle Swarm Optimization, Signals and Systems Conference, 208. (ISSC), Galway, IET Irish, pp 244-249.
Fakhimghanbarzadeh B., Hosein M. and Hossein A. (2010), Optimization of Biomass Waste Gasification Combined Heat and Power System, Electric Power and Energy Conference (EPEC), IEEE. https://doi.org/10.1109/epec.2010.5697239

Japan Institute of Energy, (2014), Asian Biomass Handbook.

Jorg Z., Thomas B-B., Oliver F., Wolfgang K. and Matin Z. (2010), Optimization of Biogas Production with Computational Intelligence A Comparative Study, Evolutionary Computation (CEC), IEEE Congress on.

Lijun W., Curtis L. W., David D. J., and Milford A. H. (2008), Contemporary issues in thermal gasification of biomass and its application to electricity and fuel production, Biomass and Bioenergy 32, pp 573-581. https://doi.org/10.1016/j.biombioe.2007.12.007

Market Report, Canada, (2016), Independent Electricity System Operator (IESO).

Michalewicz Z. (1995), A survey of constraint handling techniques in evolutionary computation methods, Proceedings of the 4th Annual Conference on Evolutionary Programming, pp. 135155.

OVO Energy Ltd.,(2017), https://www.ovoenergy.com

Sedighizadeh M., Rafiei M., and Hakimi A. (2013), Optimizing A TypicalBiomass Fueled Power Plant Using Genetic Algorithm and Binary Particle Swarm Optimization, International Journal on Technical and Physical Problems of Engineering, (IJTPE), June, No. 15, Vol. 5, 15-21.

Senjyu T., Hayashi D., Urasaki N. and Funabashi T. (2016), Optimum Configuration for Renewable Generating Systems in Residence Using Genetic Algorithm, IEEE Transactions on Energy Conversion, Vol. 21, No.2, June, 459-466. https://doi.org/10.1109/TEC.2006.874250

Twarath S. (2012), Alternative Energy Development Plan: AEDP 2012-2021, International Journal of Renewable Energy, Vol. 7, No.1.

Warsono, D. J. King, Özveren C. S. and Bradley D.A. (2007), Economic Load Dispatch Optimization of Renewable Energy in Power System Using Genetic Algorithm, PowerTech, IEEE Lausanne, 2174-2179.

Xiang W, Bayan S. S., and Oliver R. H. (2005), An Improved Resource Allocation Scheme for Plane Cove Multiple Access Using Genetic Algorithm, IEEE Transactions on Evolutionary Computation, Vol.9, No.1, February, 74-81. https://doi.org/10.1109/TEVC.2004.838662 
Yang M.-D., Chen Y.-P., Lin Y.-H., Ho Y.-F., and Lin J.-Y. (2016), Multiobjective optimization using nondominated sorting genetic algorithm-II for allocation of energy conservation and renewable energy facilities in a campus, Energy and Buildings 122, 120-130.

https://doi.org/10.1016/j.enbuild.2016.04.027

Ying-Yi H. and Ruo-Chen L. (2012), Optimal Sizing of HybridWind/PV/Diesel Generation in a Stand-Alone Power System Using Markov-Based Genetic Algorithm, IEEE Transactions on Power Delivery, Vol. 27, No. 2, April, 640647.

https://doi.org/10.1109/TPWRD.2011.2177102
Younes M., Rahli M. and Abdelhakem-Koridak L. (2007), Optimal Power Flow Based on Hybrid Genetic Algorithm, Journal of Information Science and Engineering 23, 1801-1816.

Zhang J., Chung H. S.-H., and Lo W.-L. (2007), Clustering-based adaptive crossover and mutation probabilities for genetic algorithms, IEEE Trans. Evolutionary Computation, Vol. 11, No. 3, Jun., 326-335. https://doi.org/10.1109/TEVC.2006.880727

Zhao M., Chen Z., and Blaabjerg F. (2009), Optimization of electrical system for offshore wind farms via genetic algorithm, IET Renewable Power Generation, Vol. 3, Issue. 2, June 2009, 205-216. https://doi.org/10.1049/iet-rpg:20070112

Received on 16-02-2017

Accepted on 13-05-2017

Published on 09-06-2017

DOI: https://doi.org/10.6000/1929-7092.2017.06.25

(C) 2017 Choeichum et al.; Licensee Lifescience Global.

This is an open access article licensed under the terms of the Creative Commons Attribution Non-Commercial License (http://creativecommons.org/licenses/by-nc/3.0/) which permits unrestricted, non-commercial use, distribution and reproduction in any medium, provided the work is properly cited. 